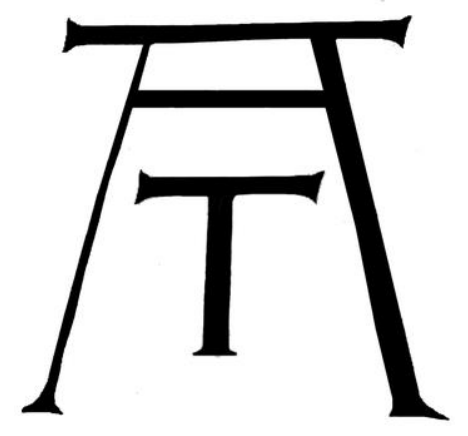

\title{
AsiaTeatro
}

rivista di studi online - ISSN: 2240-4600

www.asiateatro.it

anno 2022, fascicolo n. 1

Marilia Albanese

Gli tshechu e le danze fra terra e cielo https://doi.org/10.55154/GSDR3999 


\section{Gli tshechu e le danze fra terra e cielo}

\section{Marilia Albanese}

Nelle zone himalayane di tradizione buddhista, ovvero il regno del Bhutan e gli stati indiani del Ladakh e del Sikkim, in determinati periodi del calendario lunare si tengono ancora oggi presso i monasteri delle rappresentazioni sacre, gli tshechu, che vedono una grande partecipazione popolare. Sono particolari momenti di coesione religiosa, culturale e sociale che rinsaldano i vincoli di appartenenza alla comunità, soprattutto laddove il credo professato è minoritario. Se infatti il Bhutan è un regno di dichiarata fede buddhista, il Ladakh e il Sikkim si inseriscono in un contesto maggioritario hindu o musulmano.

La creazione di questo tipo di spettacolo si deve a Padmasambhava, il "Nato da un Loto", monaco indiano di tradizione tantrica ${ }^{1}$ che tra l'VIII e il IX secolo diffuse in Tibet la dottrina del buddhismo Vajrayāna e che è tutt'ora celebrato come Guru Rimpoche, "Venerabile Maestro", e addirittura come una forma di Buddha. Secondo la tradizione, a lui è ascritta la fondazione del primo monastero buddhista in Tibet, Samye, e la conseguente costituzione della scuola Nyingma, "Antico Ordine".

Monaco, filosofo, mistico e mago, Padmasaṃbhva racchiude in sé diversi aspetti e sicuramente la dimensione magica del suo essere è profondamente sentita in Tibet e nei paesi che oggi ne conservano la tradizione. Una delle attività maggiormente citata nelle agiografie riferite a Padmasaṃbhava è infatti la sua capacità di soggiogare spiriti maligni, demoni, orchi e soprattutto orchesse, "inchiodandoli" al suolo tramite la costruzione di edifici sacri. In tal modo le forze negative e ostili non solo venivano neutralizzate, ma addirittura convinte a servire il Buddha e i suoi seguaci. Nei cham, le danze eseguite durante gli tshechu, molti gesti rituali sono proprio volti a domare e sconfiggere le potenze oscure.

\footnotetext{
${ }^{1}$ Il Tantrismo, che prende il nome dai testi che lo espongono, i Tantra, è un sistema religioso che include pratiche rituali volte a liberare l'adepto dai normali schemi mentali per prepararlo all'incontro e all'unione con il Divino. Articolato in diverse correnti, nell'ambito buddhista si espresse nel Vajrayāna, terza evoluzione del messaggio originale del Buddha, che è alla base delle varie scuole tibetane. II Vajrayāna prende il nome da vajra, lo scettro di diamante che allude alla lucentezza e all'indistruttibilità della dottrina.
} 
Sembra che il primo tshechu si sia tenuto in Bhutan, nella valle del Bhumtang, e che in esso Padmasambhava abbia raffigurato tramite coreografie specifiche le sue otto forma di manifestazione. Il termine tshechu significa "decimo giorno" e nel decimo giorno di luna crescente di ogni mese Padmasambhava avrebbe promesso di ritornare sulla terra, per cui si tratta di una data di grande auspicio.

L'uso della maschera, fondamentale negli tshechu, non ha soltanto la funzione di raffigurare personaggi sacri noti al pubblico, ma soprattutto esercita un potere trasformativo: il monaco che la indossa si spoglia della sua individualità e del suo ego per lasciare emergere dal profondo di sé le divinità che lo abitano. Non è tanto l'interpretare un ruolo tramite il mascheramento o indurre la discesa di qualche divinità esterna, quanto piuttosto causare il risveglio delle forze già presenti nel proprio essere interiore, latenti e inattive. Il lungo e arduo training psicofisico a cui si sottopone il danzatore - sia esso monaco o laico - trasforma i suoi movimenti in gesti sacri capaci di evocare, celebrare, scongiurare, e la danza si trasforma in preghiera ispirata.

La maschera rappresenta un aspetto dell'interiorità del danzatore, ma al tempo stesso lo occulta. Tale affascinante paradosso è connesso con il termine "persona": di probabile origine etrusca, definiva la maschera teatrale e, a ben vedere, la persona è una sorta di corredo teatrale che il nostro io utilizza a seconda dei ruoli interpretati.

Le date e la durata delle celebrazioni variano da luogo a luogo e il programma non è rigidamente stabilito: si possono aggiungere e omettere brani, anche se alcuni compaiono sempre. Il teatro di rappresentazione degli tshechu è la corte del monastero e lo spazio su cui si esibiscono i danzatori è considerato un maṇạala, sacro diagramma abitato dalle diverse forze divine. I principali tshechu si tengono a Paro in Bhutan, a Hemis in Ladakh e a Gangtok nel Sikkim, ma molti altri monasteri himalayani ne organizzano annualmente.

I cham sono eseguiti sia da monaci che da laici, ma sono comunque appannaggio maschile ed anche le divinità e i personaggi mitici femminili sono interpretati da uomini; solo in un paio di casi si esibiscono anche gruppi di donne, ma si tratta di danze di intermezzo.

Lo tshechu più noto e spettacolare è quello di Paro, che si tiene nel secondo mese del calendario lunare (marzo/aprile) e dura cinque giorni. Molti dei cham che lo animano sono eseguiti anche negli altri monasteri bhutanesi, con l'aggiunta di danze diverse che celebrano avvenimenti storici e mitici legati a quel preciso luogo. 

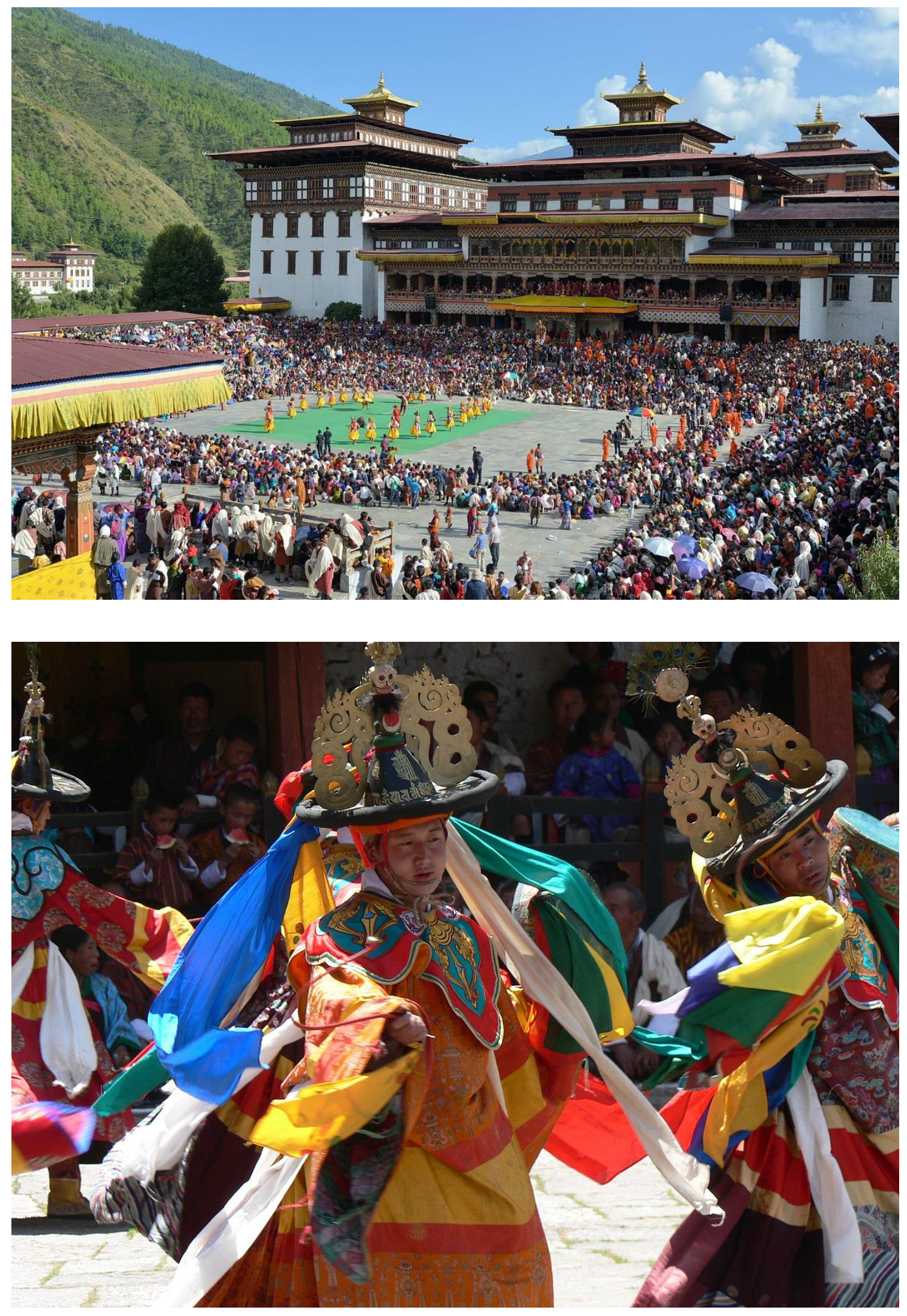
Le origini dello tsheshu di Paro risalgono al XVII sec. e il repertorio si è arricchito nel corso del tempo con l'apporto di cham creati da personaggi ispirati e da autorità politiche ${ }^{2}$. L'obbiettivo che le sacre rappresentazioni si pongono, a Paro come in tutte le altre località himalayane che mantengono ancora la tradizione dello tshechu, è propiziare le divinità perché effondano su attori e spettatori benefici spirituali. La corte del monastero diventa un mạ̣ala, ove le forze divine occupano determinati spazi, per cui le danze si sviluppano secondo precise dinamiche di movimenti. Prima, però, è necessario purificare il terreno da tutte le presenze maligne e quindi i cham di apertura dello tshechu sono apotropaici: Durdag, la danza degli Spiriti dei Luoghi di Cremazione eseguita da monaci con le maschere a teschio; Shinje YabYum, la danza di Yamantaka e della sua consorte che distruggono la Morte, ove i danzatori portano muso e corna taurine di colore rosso; Shanag, la danza dei Cappelli Neri, in cui i danzatori non sono mascherati, ma indossano ampi cappelli neri di feltro e lunghi abiti di broccato. I loro movimenti seguono le linee invisibili del maṇdala e sono volti a "uccidere" i demoni che si oppongono al Buddhismo e ai suoi devoti: l'atto violento, tuttavia, ha la funzione compassionevole di mondare gli esseri demoniaci dalla loro negatività per farli rinascere in una terra pura e incamminarli sul sentiero dell'illuminazione.

Spesso in queste danze di purificazione compare un oggetto-feticcio in cui vengono trasferite le forze malefiche, che viene trafitto con i phurba, i pugnali rituali a tre lame, o in certi casi bruciato. In Ladakh negli tshechu dei monasteri di Diskit, nella valle di Nubra, di Likir, nei pressi della capitale Leh, e nel palazzo stesso della città il momento culminante è il Dosmochhey durante il quale una grande torma ${ }^{3}$, una figura di sgargianti colori fatta di farina di orzo e burro diventata il ricettacolo delle potenze negative, viene portata in processione fuori dall'abitato e distrutta.

Le divinità terrifiche, tanto presenti nell'iconografia del buddhismo Vajrayāna, hanno sempre e comunque funzione benefica, vuoi perché distruggono i nemici del

\footnotetext{
${ }^{2}$ I mistici Dorje Lingpa e Pema Lingpa attivi nel XIV e nel XV sec. ebbero la visione di esseri celesti danzanti durante le loro pratiche meditative e ne tradussero le coreografie in particolari cham. Lo stesso fece lo Shabdrung Ngawang Namgyal, monaco tibetano grande condottiero e statista, a cui si deve nel XVII sec. I'unificazione dei vari regni locali e la fondazione di quello che oggi è il Bhutan..

${ }^{3}$ Le torma sono statuine di forma conica realizzate con un impasto base di farina d'orzo e burro di yak a cui possono essere aggiunti anche altri ingredienti; rappresentano la luna, il sole, animali, edifici, personaggi famosi in maniera stilizzata e con vivaci colori e hanno diverse funzioni. La più diffusa è quella di offerta votiva e pertanto compaiono sempre sugli altari. Possono tuttavia essere impiegate per propiziare gli spiriti e acquisire meriti. In certe cerimonie sono anche mangiate. Quando vengono usate nelle processioni, raggiungono dimensioni notevoli.
} 
dharma, la santa dottrina, vuoi perché scardinano le gabbie mentali che impediscono l'illuminazione. Nello tshechu i Tum Ngam, le danze delle Divinità Terrifiche, sono tra le più spettacolari per il campionario di maschere animali e demoniache che $\mathrm{i}$ monaci indossano su preziosi abiti di broccato. Guidati da Guru Rimpoche (Padmasambhava) nella forma di Dorji Dragpo, la "Fiera Folgore", danzano per dissolvere l'erronea concezione della dualità e celebrare il trionfo della coscienza illuminata. I Ging, armati di spade, e i Tsholing che suonano i tamburelli - entrambi i gruppi con maschere terribili - rappresentano le divinità guardiane che vegliano sul paradisiaco palazzo di Guru Padmasaṃbhava nello Zangto Pelri, la terra pura. Forza e grazia si alternano nei movimenti dei danzatori che interpretano divinità maschili e femminili, i cui ampi abiti si aprono in ventagli colorati ad ogni giro e salto.

A Thimphu, sempre in Bhutan, lo tshechu che si tiene annualmente per quattro giorni nell'ottavo mese del calendario lunare (settembre/ottobre), presenta una delle più terrificanti danze, il Lhamo Dromchoe. In essa compare Palden Lhamo, la maggiore divinità femminile del pantheon bhutanese, che agli inizi del XVIII sec. era apparsa danzando a Gyalse Kunga Gyaltshen ${ }^{4}$ durante una meditazione. Venti divinità femminili con maschere mostruose ruotano attorno a Palden Lhamo, colei che rappresenta gli innumerevoli aspetti della Grande Dea che in Bhutan è considerata la Madre di Saggezza di tutti i Buddha.

Molti brani dello tshechu, a Paro e altrove, sono rappresentazioni edificanti, come la danza del Cervo e del Cacciatore eseguita da laici, Shava Shachi, in cui il santo Milarepa $^{5}$ salva l'animale e converte il sanguinario cacciatore o ancora il Giudizio dei Morti, Raksha Mangcham, ove compaiono in scena davanti al Signore dei Morti il defunto nel suo momento di transizione - il Bardo ${ }^{6}$ - accompagnato dal Dio Bianco e dal Demone Nero, che albergano in ciascuno di noi dal primo giorno della nostra esistenza.

Il momento culminante dello tshechu è il Guru Tshengyed, la rappresentazione delle otto forme assunte da Guru Rimpoche nei vari momenti della sua vita per la diffusione del messaggio del Buddha e la difesa della sua dottrina. Si apre con una solenne

\footnotetext{
${ }^{4}$ Gyalse Kunga Gyaltshen era la reincarnazione del figlio dello Shabdrung Ngawang Namgyal.

${ }^{5}$ Jetsun Milarepa fu un famoso siddha, un "perfetto" o maestro realizzato, del XI/XII sec che ebbe vita turbolenta e che compose mistici canti ancora oggi famosi in Tibet.

${ }^{6}$ Stato liminale in cui si trova lo spirito del defunto in transito fra la morte avvenuta e la nuova incarnazione che lo attende. È descritto nel Bardo Thodol, noto in Occidente come "Libro Tibetano dei Morti "e si articola in diversi stadi con prove da superare.
} 
processione condotta da Guru Rimpoche con una maschera d'oro, seguito dalle sue otto manifestazioni, ciascuna delle quali esegue un brano di danza e si ferma accanto al Guru, che nel frattempo si è assiso sotto un baldacchino con a fianco le sue due consorti: la principessa indiana Mandarava e Yeshe Tshogyal, una khandroma ${ }^{7}$, una delle figure femminili più importanti del buddhismo tibetano. I presenti cercano di avere la benedizione del Guru e si accalcano attorno a lui, poiché il monaco che lo interpreta è ritenuto essere in quel momento il ricettacolo del Guru stesso. Durante il Guru Tshengyed ha luogo la danza delle sedici Dakini, le dee che portano sedici tipi diversi di offerte per il Guru: i danzatori indossano sui ricercati costumi speciali "grembiuli" di ossa (secondo la tradizione tantrica dovrebbero essere ossa umane) che rimandano alla transitorietà della condizione umana e alla necessità di superare la paura della morte.

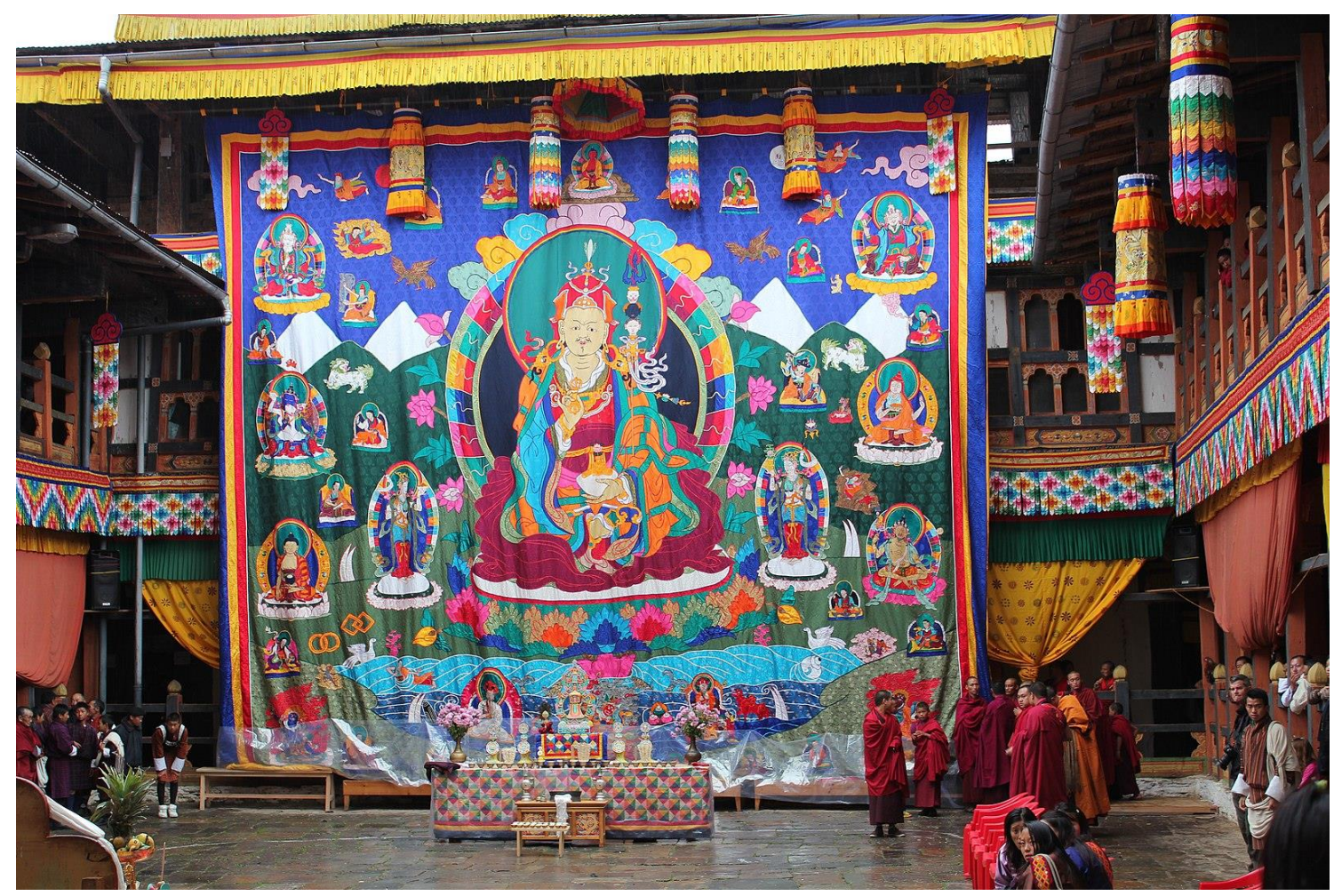

A Paro si aggiunge alle danze il Thongdrol, l'esposizione l'ultimo giorno dei quattro di celebrazioni di una colossale thangka ${ }^{8}$ di Guru Rimpoche - 30 metri per 45

\footnotetext{
7 La khandroma, "colei che si muove in cielo", è una divina figura depositaria di suprema saggezza, spesso iniziatrice di importanti personaggi nella storia del Buddhismo tibetano.

${ }^{8}$ Dipinti di soggetti sacri su stoffa, realizzati seguendo minuziose regole, che hanno diverse funzioni: descrivono e celebrano i momenti salienti della vita del Buddha, i personaggi del mondo sacro e di quello storico, particolari eventi mitici; sono il centro del culto e del cerimoniale; divengono potenti
} 
- che costituisce uno dei momenti più sacri e scenografici del festival. Il Guru vi è raffigurato con le due consorti e con le sue otto forme.Per gli spettatori lo tshechu è l'esplicitazione del loro credo e la vivente rappresentazione delle storie udite fin da bambini. Non è uno spettacolo o la drammatizzazione di un mito, è la realtà della fede e della comune matrice himalayana di quello che un tempo fu un grande popolo, oggi politicamente diviso in ladakhi, bhutanesi, sikkimesi, ma di fatto uno per cultura e storia.

Assistere allo tshechu fa acquisire meriti spirituali, ma non solo: porta buona fortuna e realizza i sogni. Sacro e profano si mescolano sulla soglia tra cielo e terra, nello spazio speciale della corte del monastero, quando le potenze divine scendono fra gli uomini.

L'accompagnamento musicale, fondamentale, è effettuato da strumenti a fiato, tra cui le grandi trombe telescopiche, il gyaling, (sorta di oboe), il kangling, (corno), la conchiglia e tonanti percussioni - tamburi, tamburelli, cembali di varia misura, campane; in certi cham si aggiunge il dramyin, un liuto a sei corde.

Alle danze sacre si alternano altre rappresentazioni: danze dei Nobili, eseguite da uomini e donne in sontuosi costumi; momenti di canto corale; interventi dei buffoni, personaggi fondamentali che irrompono nella narrazione sacra portando una nota satirica che riconduce dal cielo alla terra. Negli tshechu bhutanesi sono noti come Atsara, corruzione del termine sanscrito ācārya, che significa "maestro"; la loro funzione non è soltanto divertire, ma anche stigmatizzare situazioni sociali e politiche e usare la dissacrazione come mezzo di maggiore consapevolezza.

In alcuni tshechu la presenza di oracoli rimanda ad un sostrato animista molto più antico di quello buddhista, a cui si collega la religione Bon diffusa in Tibet, i cui rapporti con la dottrina del Buddha sono tutt'ora oggetto di controversie. In Ladakh sono famosi gli tshechu del monastero di Stock e di Matho, entrambi nei pressi di Leh, che si tengono a febbraio/marzo: a Stock gli oracoli sono due giovani laici del posto mentre a Matho sono due monaci che si preparano con particolari pratiche per tre mesi.

Per quanto eccezionale sia lo tshechu per tematiche, costumi, movenze, è inserito nel contesto del quotidiano: è una festa gioiosa ove gli spettatori sfoggiano i loro abiti migliori, consumano durante e dopo lo spettacolo i manicaretti che si sono portati, si

strumenti nel cammino spirituale quali supporti per contemplare, evocare e introiettare le divinità rappresentate. 
raggruppano con parenti e amici rinsaldando legami e intessendo relazioni matrimoniali. Certo, ci sono momenti di silenzio e venerazione, ma non si ha mai la sensazione di essere fuori dal reale, in uno spazio artefatto, su un palcoscenico. C'è poco di teatrale e molto di vitale.

Malgrado le varianti locali gli tshechu hanno uno schema comune: propiziare le forze positive e scongiurare le negative attraverso una ritualità che si avvale del linguaggio corporeo e della presenza di personaggi eccezionali evocati dai danzatori. Maschere, movenze, gesti scanditi da ritmi ancestrali sommuovono negli spettatori il mondo del profondo, offrendo loro l'emozione dell'incontro con il Sacro.

\section{Bibliografia}

ARDUSSI John, "Gyalse Tenzin Rabgye and the Founding of Taktsang Lhakhang", Journal of Bhutan Studies, vol. 1, no.1. (1999), Thimphu

ARDUSSI John and Sonam TOBGYE, "Gyalse Tenzin Rabgye and the Celebration of Tshechu in Bhutan", Written Treasures of Bhutan. Mirror of the Past and Bridge to the Future, vol II Thimphu, Bhutan, National Library, 2008

ARDUSSI John, "Gyalse Tenzin Rabgye and the Founding of Tshechu in Bhutan”, Written Treasures of Bhutan, Thimphu, Bhutan, National Library, 2009

BARTHOLOMEW Therese Tse, JOHNSTON John (a cura di), Au pays $d u$ Dragon. Arts sacrés du Bhoutan, Annexe V, Reunion des Musees Nationaux, 2009

KHENPO Phuntshok Tashi, Invoking Happiness. Guide to the sacred festivals of Bhutan \& Gross National Happiness, 2011 